\title{
Evaluación del comportamiento agronómico de 15 líneas de maní del grupo Valencia (Arachis Hypogaea L.) (ㄷ)(1)(2)
}

\section{Evaluation of the agronomic performance of 15 lines of mani of the group Valencia (Arachis hypogaea L.)}

M.Sc, Sixto Santiago Ibáñez Jácome. 1, MAE. Evelyn Eugenia Alcívar Soria. 2, MAE. Lenin Landívar Cedeño Alcívar. 3, M.Sc. Julio Cesar Caicedo Aldaz.4

Recibido: 02-09-2017 / Revisado:03-11-2017 Aceptado: 10-12-2017/ Publicado: 01-01-2018

\begin{abstract}
.
DOI: https://doi.org/10.33262/cienciadigital.v2i1.18

The Peanut, is another important source of vegetables oil in tropical and subtropical area of the planet. It is native to the tropical South America, probably Brazil. Al though some Asian Countries, mainly Chinese e Indian, produce about two - thirds of the global harvest, in the actuality the peanut is one source important the cooking oil in the American Tropics, ranking second over oil palm in Africa in our country, groundnut areas are located in the provinces of Manabí, Loja and El Oro, where are grown from 10.000 to 15.000 has; with average yields of $800 \mathrm{~kg} \mathrm{ha}^{-1} \mathrm{de}$ peanut shell. This production compared with the genetic potential of improved varieties is low and, Is mainly due to the lack of improved varieties available, poor quality seeds, inadequate management of the crop, presence of insect pests diseases, due to high demand of peanut kernels, the INIAP through oilseed program short cycle maintains a continuous process of research in peanut producing areas, to generate new materials with higher yield potential, resistance or tolerance to insect pests, diseases and better grain quality, for different market segments, in this research, the following objectives were i)
\end{abstract}

\footnotetext{
1Universidad Técnica Luis Vargas Torres de Esmeraldas Ext. la Concordia, chisanty1@ @otmail.com 2Universidad Técnica Luis Vargas Torres de Esmeraldas Ext. la Concordia, evelyn.alcivar@utelvt.edu.ec 3Universidad Técnica Luis Vargas Torres de Esmeraldas Ext. 1a Concordia, leninced62@ hotmail.com 4Universidad Técnica Luis Vargas Torres de Esmeraldas Ext. la Concordia, juliocesarcaicedo@hotmail.com
} 
Evaluate the agronomic and morphological 15 peanut cultivars Valencia in Group Farm agroecological zone in Caluma the Triumph. ii)Select the line with the best agronomic and morphological characteristics for this agro-ecological zone, and iii) Establish a database of type Valencia peanut germplasm to continue the research process. The experimental design was Randomized Complete Block (BCA) with 15 treatments and 3 replications: e performed variance analysis, Tukey test at 5\% to compare means of treatments and correction analysis and linear regression. The peanut germplasm response in terms of yield components evaluated in this agro-ecological zone were very different, except for the percentage of seeds by vainas. Los Vaneamiento and higher average yields peanuts, were recorded in treatments T6: Pedro Carbo with 5,456 and T13 (RCM - 112) with $5,053 \mathrm{~kg} / \mathrm{ha}$ at $14 \%$ moisture. The yield component contributing to increase peanut yield was the percentage of grain with $12 \%$. The highest number of pods per plant was evaluated in the T8 (Saavedra Pearl) with 20 pods. The percentage of grain (PG) highest was recorded in T5 (Bowling), with $88.33 \%$ due to the sheath and grain quality. Selected lines to continue the research process in this area in the agro-ecological zone Caluma are: T6: Pedro Carbo, T13: RCM - 112, T4 PI26202301-5 D and T12 SPZ - 457. Finally this research helped generate valuable information and results to improve peanut production in agro-ecological zone Farm Triumph, Caluma.

Keywords: Agronomy, Agricultural Production, Productivity, Agricultural Área

\section{Resumen.}

El maní, es otra importante fuente de aceite vegetal en las zonas tropicales y subtropicales del planeta. Es nativo de la parte tropical de América del Sur, probablemente del Brasil. Aun cuando algunos países asiáticos, principalmente China e India, producen cerca de las dos terceras partes de la cosecha mundial, en la actualidad el maní es una fuente importante de aceite para cocinar en los trópicos americanos, ocupando el segundo lugar respecto a la palma de aceite en África. En nuestro país, las zonas maniseras están localizadas en las provincias de Manabí, Loja y El Oro, en donde se cultivan de 10.000 a 15.000 has; con rendimientos promedios de $800 \mathrm{~kg} \mathrm{ha}^{-1} \mathrm{de}$ maní 
en cáscara. Esta producción comparada con el potencial genético de las variedades mejoradas es baja y, se debe principalmente a la falta de variedades mejoradas disponibles, semillas de mala calidad, manejo inadecuado del cultivo, presencia de insectos plagas y enfermedades. Debido a la alta demanda del grano de maní, el INIAP a través del Programa de Oleaginosas de Ciclo Corto mantiene un proceso continuo de investigación en las zonas productoras de maní, para generar nuevos materiales con mayor potencial de rendimiento, resistencia o tolerancia a insectos plagas, enfermedades y mejor calidad del grano, para los diferentes segmentos del mercado. En esta investigación se plantearon los siguientes objetivos evaluar las características agronómicas y morfológicas de 15 cultivares de maní del grupo Valencia en la zona agroecológica de la Granja el Triunfo en Caluma. Que además nos permita seleccionar las líneas con las mejores características agronómicas y morfológicas para esta zona agroecológica, y Establecer una base de datos de germoplasma de maní tipo Valencia para continuar con el proceso de investigación. Se utilizó un diseño de Bloques Completos al Azar (BCA), con 15 tratamientos y 3 repeticiones: Se realizó el Análisis de varianza, Prueba de Tukey al $5 \%$ para comparar promedios de tratamientos $\mathbf{y}$ Análisis de corrección y regresión lineal. La respuesta del germoplasma de maní en cuanto a los componentes del rendimiento evaluado en esta zona a agroecológica fue muy diferente, con excepción del porcentaje del Vaneamiento y Semillas por Vainas. Los rendimientos promedios más altos de maní, se registraron en los tratamientos T6: Pedro Carbo con 5.456 y el T13 (RCM - 112) con $5.053 \mathrm{~kg} / \mathrm{ha}$ al $14 \%$ de humedad. El componente del rendimiento que contribuyó a incrementar el rendimiento de maní fue el porcentaje de grano con el 12\%. El mayor número de vainas por planta se evaluó en el T8 (Perla de saavedra), con 20 vainas. El porcentaje de grano (PG) más alto, se registró en el T5 (Boliche), con el 88,33 \% debido a la calidad de vaina y grano. Las líneas seleccionadas para continuar con el proceso de investigación en este rubro en la zona agroecológica de Caluma son: T6: Pedro Carbo, T13: RCM - 112, T4 PI26202301 - 5 D y T12 SPZ - 457. Finalmente, esta investigación contribuyó a generar información y resultados valiosos para mejorar la producción de maní en la zona agroecológica de la Granja el Triunfo, Caluma. 
Palabras Claves: Agronomía, Producción Agrícola, Productividad, Zona Agrícola.

\section{Introducción.}

El maní, es otra importante fuente de aceite vegetal en las zonas tropicales y subtropicales del planeta. Es nativo de la parte tropical de América del Sur, probablemente del Brasil. Aun cuando algunos países asiáticos, principalmente China e India, producen cerca de las dos terceras partes de la cosecha mundial. En la actualidad el maní es una fuente importante de aceite para cocinar en los trópicos americanos, ocupando el segundo lugar respecto a la palma de aceite en África. (Guiller, P. y Silvestre, P. 2000)

El maní se siembra aproximadamente en 20 millones de has y ocupa el tercer lugar entre las leguminosas de grano, después de la soya y el frijol común, los cuales se producen en 55 y 26 millones de has en el mundo. Los principales países que cultivan maní a nivel mundial son India, China y EE.UU, cuya producción mostró un incremento en los últimos años. El maní tiene demanda para el consumo directo, luego de tostado.

En nuestro país, las zonas maniseras, están localizadas en las provincias de Manabí, Loja y El Oro, en donde se cultivan de 10.000 a 15.000 has; con rendimientos promedios de $800 \mathrm{~kg}$ ha $^{-1}$ de maní en cáscara. Esta producción comparada con el potencial genético de las variedades mejoradas es baja y, se debe principalmente a la falta de variedades mejoradas disponibles, semillas de mala calidad, manejo inadecuado del cultivo.

En esta investigación se plantearon los siguientes objetivos:

- Evaluar las características agronómicas y morfológicas de 15 cultivares de maní del grupo Valencia en la zona agroecológica de la Granja el Triunfo en Caluma.

- Seleccionar las líneas con las mejores características agronómicas y morfológicas para esta zona agroecológica.

- Establecer una base de datos de germoplasma de maní tipo Valencia para continuar con el proceso de investigación.

\section{Metodología.}

Para la presente investigación descriptiva se utilizó el método teórico - científico y estadístico nos permitió según los objetivos apoyarnos de los métodos cualitativos y cuantitativos, Para la contaminación por glifosato de los suelos, se realizó un análisis completo (físico, químico, y biológico), con el cual se determinó que camino se debía seguir para solucionar el problema planteado.

Para desarrollar la investigación la metodología y procedimientos fueron los siguientes, mismos que iniciaron con la selección de los lotes a muestrear.

Se estudiaron 15 líneas promisorias de maní, tipo Valencia procedentes del Programa de Oleaginosas del INIAP. EELS. 


\section{Tratamientos.}

TABLA 1. SE CONSIDERÓ UN TRATAMIENTO A CADA LÍNEA DE MANÍ SEGÚN EL SIGUIENTE DETALLE

\begin{tabular}{|c|c|}
\hline Tratamientos $\mathbf{N}^{\circ}$ & Código \\
\hline $\mathrm{TI}$ & MB-645 \\
\hline $\mathrm{T} 2$ & Y-714 \\
\hline T3 & CHARAPOTO \\
\hline $\mathrm{T} 4$ & PI-26202301-5D \\
\hline T5 & BOLICHE \\
\hline T6 & PEDRO CARBO \\
\hline $\mathrm{T7}$ & CRIOLLO LOJA \\
\hline T8 & PERLA DE SAAVEDRA \\
\hline T9 & FLOR RUNNER NEMATOL \\
\hline $\mathrm{T} 10$ & SANGRE CRISTO \\
\hline $\mathrm{T} 11$ & RCM 207 \\
\hline $\mathrm{T} 12$ & SPZ-457 \\
\hline T13 & RCM-112 \\
\hline $\mathrm{T} 14$ & INIAP 380 \\
\hline T15 & INIAP 381 \\
\hline
\end{tabular}

Fuente: INIAP.2012. 


\section{Procedimiento}

Se utilizó un diseño de Bloques Completos al Azar (BCA).

Número de localidades: 1

Número de tratamientos: $\quad 15$

Número de repeticiones: 3

Número de unidades experimentales: $\quad 45$

Área total de la unidad investigativa: $\quad 5 \mathrm{~m} \mathrm{x} 1,8 \mathrm{~m}=9 \mathrm{~m}^{2}$

Área total del ensayo: $\quad 9 \mathrm{~m}^{2} \mathrm{x} 45 \mathrm{ue}=405 \mathrm{~m}^{2}$

Área neta de la unidad investigativa: $\quad 4 \mathrm{~m} \times 0.9 \mathrm{~m}=3.6 \mathrm{~m}^{2}$

Área total del ensayo con caminos: $\quad 18 \mathrm{~m} \times 27 \mathrm{~m}=486 \mathrm{~m}^{2}$

Distancia entre hileras: $\quad 0,45 \mathrm{~m}$

Número de hileras por unidad investigativa: $\quad 4$

Numero de hileras por unidad neta investigativa: 2

\section{Tipos de análisis.}

TABla 2. ANÁlisis DE VARIANZA (ADEVA) SEGÚN EL SIGUIENTE DETALLE

\begin{tabular}{lcc}
\hline \multicolumn{1}{c}{ FUENTES DE VARIACIÓN } & GRADOS DE LIBERTAD & CME* \\
\hline Bloques (r-1) & 2 & $\int^{2} e+15 \int^{2}$ bloques \\
Líneas (t-1) & 14 & $\int^{2} e+3 \theta^{2} \mathrm{t}$ \\
Error Experimental (t-1) (r-1) & 28 & $\int^{2} e$ \\
Total (txr)-1 & 44 & \\
\end{tabular}

Cuadrados Medios Esperados. Modelo fijo. Tratamientos seleccionados por el investigador.

- Prueba de Tukey al $5 \%$ para comparar promedios de tratamientos en las variables que el Fisher Calculado fue significativa.

- Análisis de corrección y regresión lineal. 


\title{
Métodos de evaluación y datos tomados.
}

\section{Porcentaje de germinación en laboratorio (PGL).}

Se realizó 15 días antes de la siembra en una muestra de 100 semillas de cada accesión, en bandejas de plástico con papel filtro. Se contaron el número de semillas germinadas y se expresó en porcentaje.

\section{Días a la emergencia (DE).}

Esta variable se registró en días transcurridos desde la siembra y hasta cuando más del 50\% de plántulas emergieron en la parcela total.

\section{Porcentaje de emergencia en el campo (PEC).}

En un período de tiempo comprendido entre los 10 a 20 días después de la siembra; se contaron las plantas emergidas en la parcela neta; y se expresó en porcentaje de acuerdo con el número de semillas sembradas por parcela.

\section{Días a la floración (DF).}

Variable que se registró contando los días transcurridos desde la siembra hasta cuando más del $50 \%$ de las plantas de cada parcela neta presentaron flores abiertas.

\section{Adaptación vegetativa (vigor) (AV).}

La evaluación de esta variable se realizó en toda la parcela, cuando las plantas estuvieron en floración, observando a simple vista el estado fenotípico de cada tratamiento, se utilizó la siguiente escala:
1: Excelente.
3: Buena.
5: Intermedia.
7: Pobre.
9: Muy pobre.

Fuente: INIAP. 2004.

\begin{abstract}
Altura de planta en (cm) (AP).
La altura de planta se midió en cm con la ayuda de un flexómetro, considerando desde el nivel del suelo hasta la yema apical del tallo principal. La altura de planta se midió en 10 plantas tomadas al azar del área neta de cada parcela en la fase de madurez fisiológica.
\end{abstract}




\section{Ramas por planta (RP).}

En 10 plantas tomadas al azar de la parcela neta de cada tratamiento, se contaron el número de ramas por planta, y se calculó un promedio. Esta variable se evaluó en la madurez fisiológica.

\section{Días a la cosecha (DC).}

Se registró el número de días comprendido desde la siembra hasta la cosecha y se consideró cuando las paredes internas de las vainas (cáscara) presentaron un color café oscuro.

\section{Vainas por planta (VP).}

En 10 plantas tomadas al azar del área útil, se procedió a arrancar las plantas y contar el número total de las vainas por planta.

\section{Vaneamiento (V\%).}

Después de la cosecha se contaron 100 vainas, tomadas al azar del área neta, luego se seleccionaron las vainas vanas, para expresar en porcentaje.

\section{Semilla por planta (SP).}

Se contaron el número de semillas por planta en el momento de la cosecha y trilla.

\section{Semilla por vaina (SV).}

Se obtuvo dividiendo el número de semillas por planta sobre el número de vainas después de la cosecha del maní en cada unidad experimental.

\section{Peso de 100 semillas (g) (PS).}

Se contaron 100 semillas tomadas al azar de cada tratamiento, luego se pesó en una balanza de precisión en gramos.

\section{Porcentaje de grano (PG).}

El porcentaje de grano se realizó después de la cosecha, utilizando la siguiente fórmula:

$$
\mathrm{D}=\ldots \text { - }-\ldots \times 100
$$

$\mathrm{P} 2$

$\mathrm{P}_{1}=$ Peso uno: vainas (cáscara) más grano.

$\mathrm{P}_{2}=$ Peso dos: grano. Fuente: (Monar, C. 2000). 


\section{Porcentaje de humedad del grano (PH).}

Este indicador de humedad, se evaluó con la ayuda de un determinador portátil de humedad en porcentaje, después de la cosecha en una muestra de cada unidad experimental, en el laboratorio del INIAP-EELS.

\section{Rendimiento por parcela (RP).}

Una vez que se cosechó y se rompió el cuesco que protege a la semilla del maní de cada parcela neta, se pesó en una balanza de reloj en $\mathrm{kg} / \mathrm{parcela}$.

\section{Rendimiento (kg/ha) (RH).}

El rendimiento en $\mathrm{Kg} / \mathrm{ha}$, se calculó utilizando la siguiente fórmula:

$$
\begin{aligned}
& 10.000 \mathrm{~m}^{2} / \mathrm{ha} \quad 100-\mathrm{HC}
\end{aligned}
$$

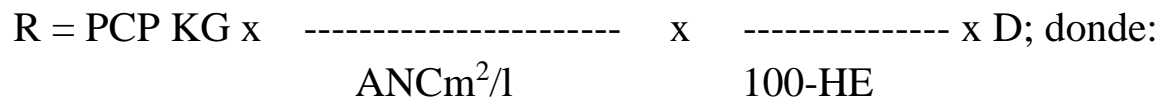

$\mathrm{R}=$ Rendimiento en $\mathrm{Kg} . / \mathrm{ha}$, al $14 \%$ de humedad

$\mathrm{PCP}=$ Peso de campo por parcela en $\mathrm{Kg}$

ANC = Área neta cosechada en $\mathrm{m}^{2}$

$\mathrm{HC}=$ Humedad de cosecha en porcentaje

$\mathrm{HE} \quad=$ Humedad estándar (14\%).

$\mathrm{D} \quad=$ Porcentaje de grano de maní

Fuente: (Monar, C. 2000).

\section{Resultados y discusión.}

\section{Variables agronómicas.}

Cuadro $\mathbf{N}^{\mathbf{0}}$ 1. Resultados de la prueba de Tukey al $5 \%$ en las variables que presentaron diferencia estadística significativa para comparar los promedios de los tratamientos en las variables agronómicas: Porcentaje de germinación en laboratorio (PGL), Días a la emergencia (DE), Porcentaje de emergencia en el campo (PEC), Días a la floración (DF), Altura de planta (AP), Ramas por planta (RP), Días a la cosecha (DC), Vainas por planta (VP), Vaneamiento (V\%), Semillas por planta (SP), Semillas por vaina (SV), Peso de 100 semillas (g) (PS), Porcentaje de grano (PG), y Rendimiento en $\mathrm{kg} / \mathrm{ha}(\mathrm{RH})$ al $14 \%$ de humedad . Caluma. 2013. 
TABLA 3. RESULTADOS DE LA PRUEBA DE TUKEY

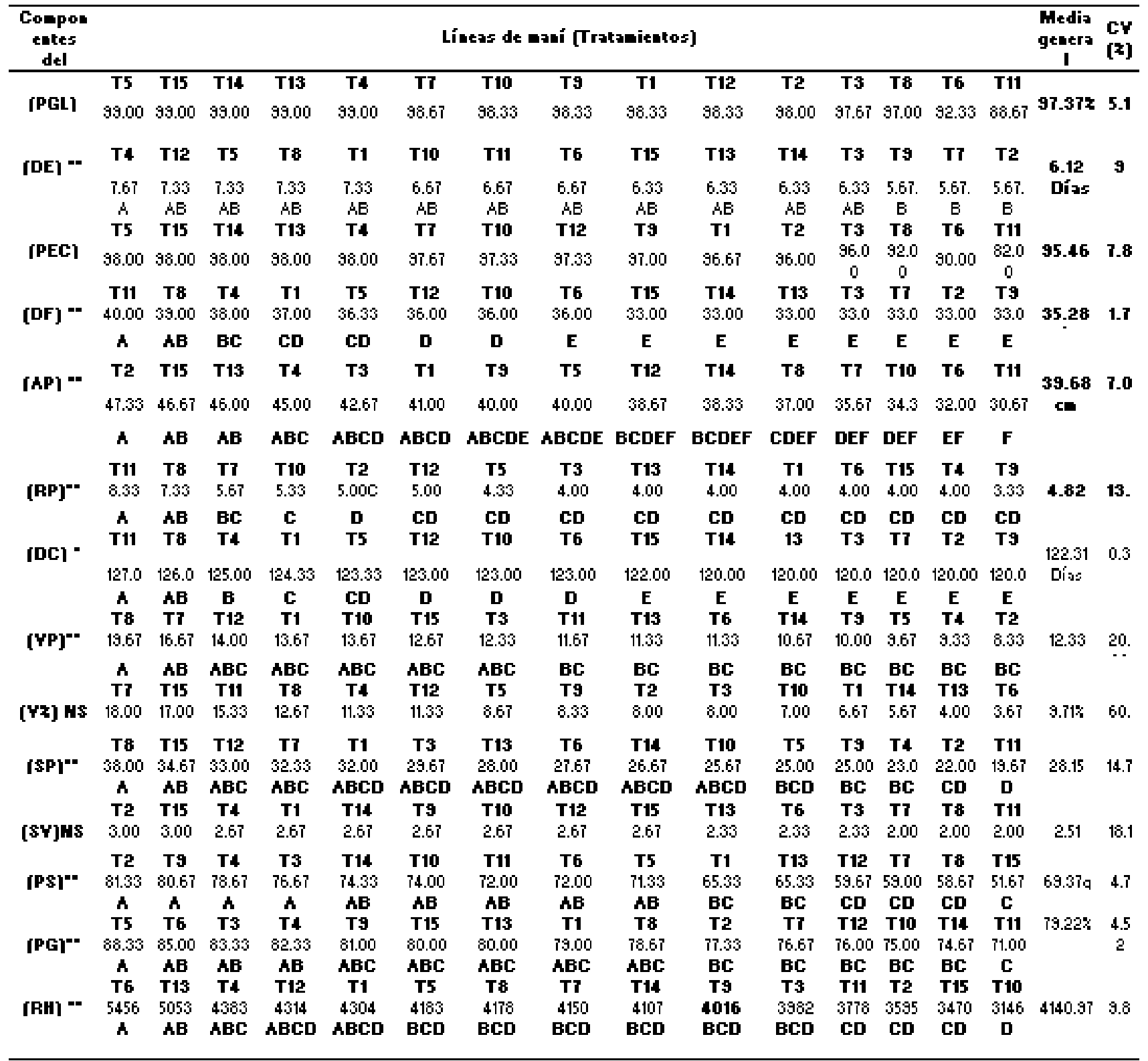

Promedios con la misma letra son estadísticamente iguales al $5 \%$, promedios con distintas letras son estadísticamente diferentes al 5\$

\section{Tratamientos (Líneas de Maní).}

La respuesta de las diferentes líneas en cuanto a las variables agronómicas: Días a la emergencia (DE), Días a la floración (DF), Altura de planta (AP), Ramas por planta (RP), Días a la cosecha (DC), Vainas por planta (VP), Semillas por planta (SP), Peso de 100 semillas (g) (PS ), Porcentaje de grano (PG), y Rendimiento en $(\mathrm{kg} / \mathrm{ha})(\mathrm{RH})$, fueron diferentes (Cuadro $\mathrm{N}^{\circ}$ 
1). Sin embargo en las variables: Porcentaje de germinación en laboratorio (PGL), Porcentaje de emergencia en el campo (PEC), Vaneamiento (V\%), y Semillas por vaina (SV), fueron estadísticamente similares (NS) (Cuadro $\mathrm{N}^{\mathrm{o}} 1$ ).

En la variable Días a la emergencia (DE), se determinó que el promedio más alto se registró en el T4 (PI-26202301-5D) con 7,67 (8) días y el más precoz fue el T2 (Y-714) con 5,67 (6) días. (Cuadro $\left.\mathrm{N}^{\mathrm{o}} 1\right)$.

Esta variable (DE) dependió de otros factores determinantes como calidad y cantidad de semilla, profundidad de siembra, humedad, $\mathrm{O}_{2}, \mathrm{CO}_{2}$ y la temperatura.

En la variable Días a la floración (DF), se registró un promedio de 40 días en el T11 (RCM 207), y la línea más precoz fue en el T9 (Flor runner nematol), con 33 días. (Cuadro $\mathrm{N}^{\circ} 1$ )

Los factores determinantes en la variable (DF), son las condiciones bioclimáticas, altitud, temperatura, humedad, cantidad y calidad de luz solar.

La variable (DF), es una característica varietal y dependen de su interacción genotipo - ambiente.

En la variable Altura de planta en (cm) (AP), el promedio más alto se evaluó en el T2 (Y-714), con $47,33 \mathrm{~cm}$, y el menor en el T9 (Flor runner nematol), con 30,67 cm (Cuadro $\mathrm{N}^{\mathrm{o}} 1$ ).

Estos resultados se dieron por la fertilidad del suelo y además hubo otros factores determinantes como la cantidad de luz solar, humedad y la asimilación de nutrientes por la planta.

Además, la (AP), es una característica de cada línea de maní y depende de su interacción genotipoambiente (Gráfico $\mathrm{N}^{\mathrm{o}} 1$ )

Gráfico $\mathbf{N}^{0}$ 1. Altura de planta en $\mathrm{cm}$.

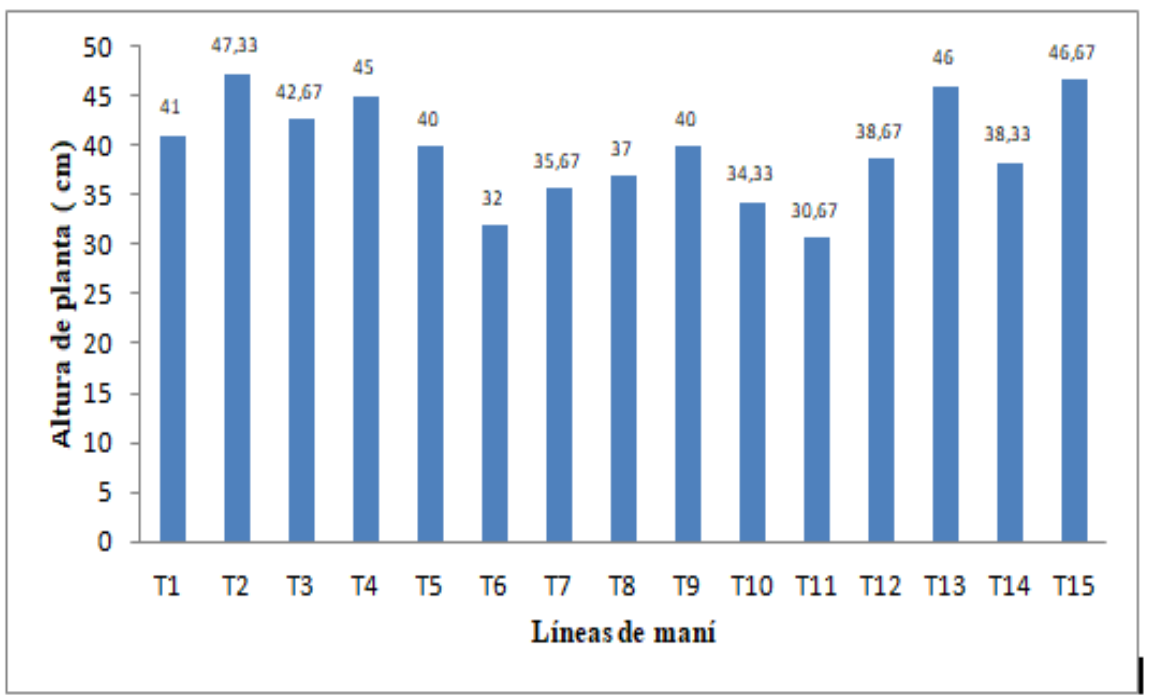

Elaborado por: Grupo de Investigación. 
Para la variable Ramas por planta (RP), el promedio más alto se dio en el T11 (RCM 207), con 8 ramas por planta y el menor número se registró en el T9 (Flor runner nematol), con 3 ramas por planta, (Cuadro $\left.\mathrm{N}^{\circ} 1\right)$.

Esta variable depende mucho de la genética de cada línea y su interacción genotipo-ambiente.

Otros factores que influyeron en las (RP), quizá fueron la cantidad y distribución de la recipitación, la densidad poblacional, el hábito de crecimiento, etc.

En el componente Días a la cosecha (DC), la línea más tardía fue el T11 (RCM 207), con 127 días y el más precoz el T9 (Flor runner nematol) con 120 días. (Cuadro No 1).

Esta diferencia se dio principalmente debido a las características de cada línea e interacción genotipo - ambiente.

Son determinantes también la temperatura, humedad, textura del suelo, calidad y cantidad de luz solar, viento, fotoperíodo, etc. $\left(\right.$ Gráfico $\left.\mathrm{N}^{\mathrm{0}} 2\right)$

Gráfico No 2. Días a la cosecha.

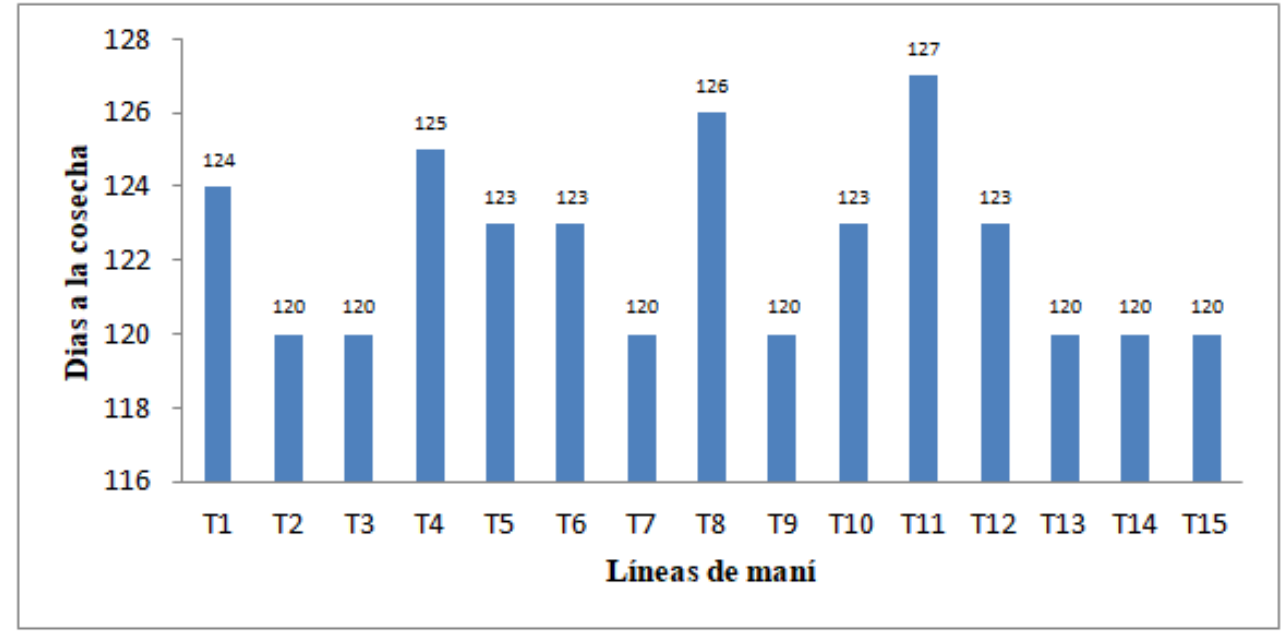

Elaborado por: Grupo de Investigación.

Para la variable Vainas por planta (VP), el promedio más alto se registró en el T8 (Perla de saavedra), con 20 vainas y el promedio más bajo en el T2 (Y-714), con 8 vainas. (Cuadro No 1).

Esta variable depende de la genética de la planta, como también las condiciones climáticas y también la fertilidad del suelo donde se desarrolla el cultivo. (Gráfico №3) 
Gráfico No 3. Vainas por planta.

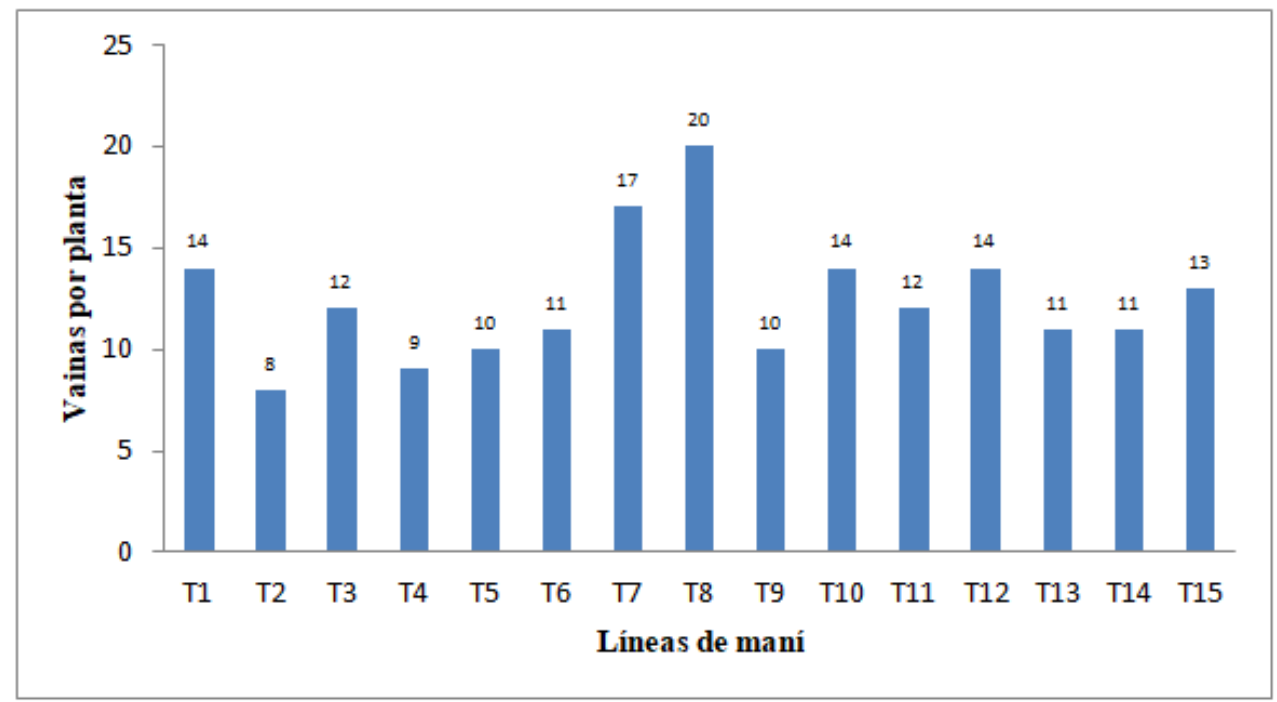

Elaborado por: Grupo de Investigación.

En la variable Semillas por planta (SP), el promedio más alto, se evaluó en la línea T8 (Perla de saavedra), con 38 semillas por planta y el menor número de semillas en el T11 (RCM 207), con 20 semillas por planta. (Cuadro $\mathrm{N}^{\mathrm{o}} 1$ ).

Las semillas por planta dependen de los caracteres de cada línea y dependen de su interacción genotipo ambiente; como también el manejo agronómico del cultivo y las buenas prácticas de manejo, como la sanidad del cultivo, la cantidad y distribución de la lluvia, etc.

En cuanto a la variable Peso de 100 semillas (g) (PS), se determinó el promedio más alto en el T2 (Y-714), con 81,33 gramos y el menor en el T15 (INIAP 381), con 51,57 gramos. (Cuadro $\mathrm{N}^{\mathrm{o}} 1$ ).

En promedio general el T2 (Y-714), registró 29,76 gramos más en relación al T5 (INIAP 381), quizá por ser un carácter de cada línea y una fuerte interacción genotipo-ambiente. El (PS), depende también del tamaño y contenido de humedad del grano.

Otros factores que incidieron en este carácter de cada línea fueron el tamaño y sanidad del grano, temperatura, humedad del suelo, cantidad y calidad de luz solar, fotoperiodo, índice de área foliar, número de granos por vaina y calidad del grano. 
En la variable Porcentaje de grano (PG), el promedio más alto se registró en el T5 (Boliche), con $88,33 \%$ y el menor en el T11 (RCM 207), con $71 \%$. (Cuadro $\left.\mathrm{N}^{\mathrm{o}} 1\right)$

En cuanto a esta variable los componentes determinantes para que el porcentaje de grano sea mayor, dependen de la calidad de vaina y grano de las diferentes líneas de maní como también las condiciones climáticas de la zona agroecológica. (Gráfico $\mathrm{N}^{\circ} 4$ )

Gráfico No 4. Porcentaje de grano.

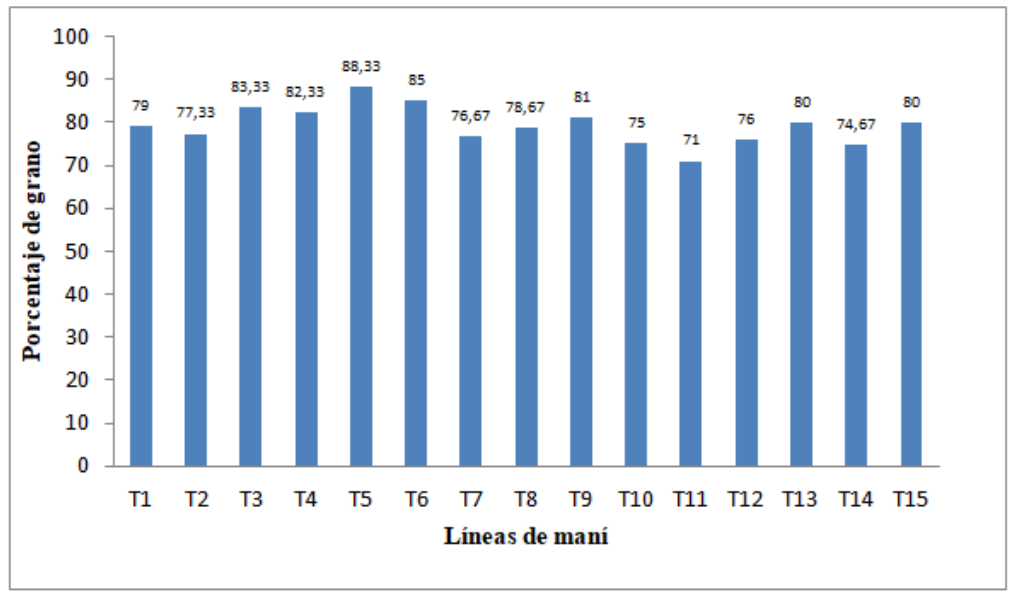

Elaborado por: Grupo de Investigación

Para la variable Rendimiento en (kg/ha) $(\mathbf{R H})$, el tratamiento con el promedio más alto fue el T6 (Pedro carbo), con $5456 \mathrm{~kg} / \mathrm{ha}$, y el de menor promedio fue el T10 (Sangre de cristo) con $3146 \mathrm{~kg} / \mathrm{ha}$ (Cuadro No 1$)$.

También es determinante sobre todo cuando las condiciones climáticas y de manejo, son adecuadas para el cultivo.

Otros factores que inciden en este carácter de cada línea son el tamaño del grano, sanidad del grano, temperatura, humedad del suelo, cantidad y calidad de luz solar, fotoperíodo, altitud, índice de área foliar, tasa de fotosíntesis, longitud de vaina, número de granos por vaina, peso de 100 semillas, calidad del grano, sanidad y nutrición de las plantas. (Gráfico Nº 3) 
Gráfico No 5. Rendimiento en kilogramos por hectárea $(\mathrm{RH})$

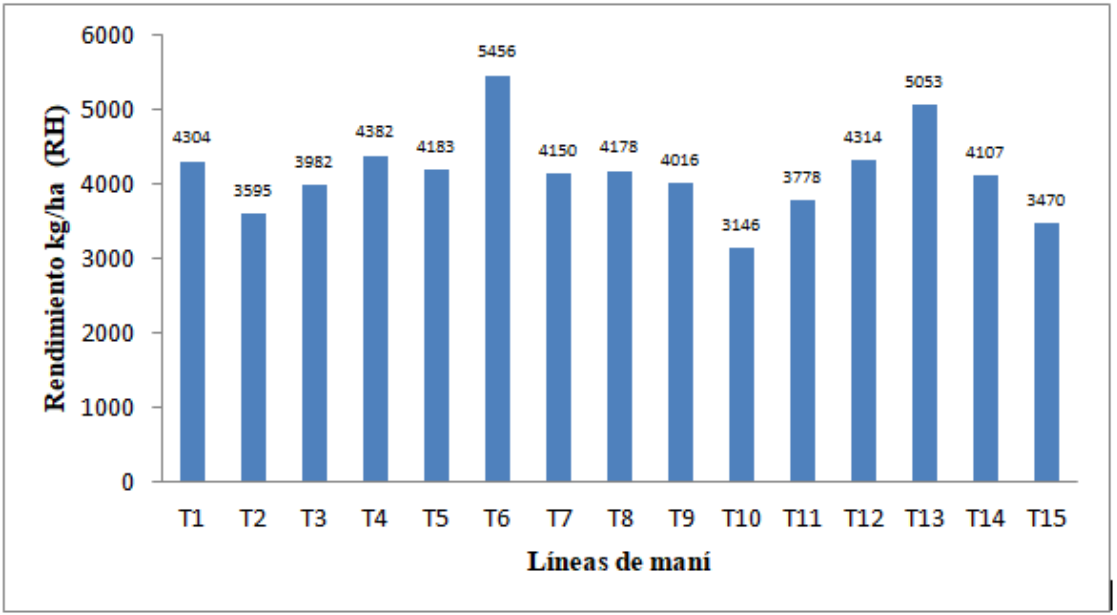

Elaborado por: Grupo de Investigación

En cuanto a las variables Porcentaje de germinación en el laboratorio (PGL), Vaneamiento (V\%), y Semillas por vaina (SV), tuvo el germoplasma una respuesta similar, con una media general del 97,37\% de germinación; es decir una buena calidad de semilla y vigor; con $9.71 \%$ de vainas vanas y 2.51 (3) semillas por vaina (cuadro $\mathrm{N}^{\mathrm{o}} 1$ ). Estos indicadores están dentro del rango reportado por varios autores en estudios en otras zonas agroecológicas maniseras del Ecuador.

\section{Coeficiente de variación (cv).}

El cv, es un indicador estadístico, que nos indica la variabilidad de los resultados y se expresa en porcentaje.

Varios autores como Beaver, J. y Beaver, L.; manifiestan que en variables que están bajo el control del investigador, deben ser valores inferiores al $20 \%$ el CV.

En esta investigación se calcularon valores del CV muy inferiores al $20 \%$ en las variables agronómicas: Porcentaje de germinación en laboratorio (PGL), Porcentaje de emergencia en el campo (PEC), Días a la emergencia (DE), Días a la floración (DF), Altura de planta (AP), Ramas por planta (RP), Días a la cosecha (DC), Semillas por planta (SP), Semillas por vainas (SV), Peso de 100 semillas (PS), Porcentaje de grano 
(PG), Rendimiento en (kg/ha) (RH) que estuvieron bajo el control del investigador por lo tanto las inferencias, conclusiones y recomendaciones son válidas para esta zona agroecológica.

Se calculó un valor superior al $20 \%$ del CV en la variable Vaneamiento (V\%) porque no depende del control del investigador y depende de su interacción genotipo-ambiente y particularmente de las condiciones bioclimáticas, como la humedad, precipitación, temperatura y la adaptación en esta zona agroecológica.

\section{Análisis de correlación y regresión lineal.}

Resultados del análisis de correlación y regresión lineal de las variables independientes (Xs) que tuvieron una relación estadística significativa con el rendimiento del cultivo de maní con un $13 \%$ (Variable Dependiente Y).

TABLA 4. RESULTADOS DEL ANÁLISIS DE CORRELACIÓN Y REGRESIÓN LINEAL DE LAS VARIABLES INDEPENDIENTES (XS)

\begin{tabular}{lccc}
\hline $\begin{array}{c}\text { Variables e indicadores } \\
\text { independientes (Xs) } \\
\text { (Componentes de } \\
\text { rendimiento) }\end{array}$ & $\begin{array}{c}\text { Coeficiente de } \\
\text { correlación } \\
\text { "r" }\end{array}$ & $\begin{array}{c}\text { Coeficiente de } \\
\text { regresión "b" }\end{array}$ & $\begin{array}{c}\text { Coeficiente de } \\
\text { determinación (R2) (\%) }\end{array}$ \\
\hline Porcentaje de grano (PG) & $0,346^{*}$ & $48,110^{*}$ & 12 \\
Vaneamiento(V \%) & $-0,443^{*}$ & $-44,954^{*}$ & 20 \\
\hline
\end{tabular}

Elaborado por: Grupo de Investigación.

$*=$ Significativo al $5 \%$.

$* *=$ Altamente significativo al $1 \%$.

\section{Coeficiente de correlación " $r$ ".}

Correlación en su concepto más simple, es la relación positiva o negativa entre dos variables y su valor máximo es +/-1 y no tiene unidades. (Monar, C. 2010)

En esta investigación las variables independientes que presentaron una correlación significativa positiva con el rendimiento fue el Porcentaje de grano. (Cuadro $\mathrm{N}^{\circ}$ 2) y la 
variable que presentó una estrechez significativa negativa con el rendimiento fue el \% de Vaneamiento (Cuadro $\mathrm{N}^{\mathrm{o}} 2$ ).

\section{Coeficiente de regresión "b".}

El coeficiente de regresión es la asociación positiva o negativa entre las variables independientes (Xs) versus el rendimiento o variable dependiente (Y). Dicho de otra manera es el incremento o disminución del rendimiento en $\mathrm{Kg} / \mathrm{ha}$; por cada cambio único de la (s) variable(s) independiente(s). (Monar, C. 2010).

En esta investigación la variable independiente que contribuyó a aumentar el rendimiento de maní evaluado en $\mathrm{Kg} / \mathrm{ha}$ fue el Porcentaje de grano. (Cuadro $\mathrm{N}^{\circ}$ 2) y la variable que redujo el rendimiento fue el porcentaje de vaneamiento (Cuadro $\mathrm{N}^{\circ} 2$ ).

\section{Coeficiente de determinación $\left(\mathbf{R}^{2}\right)$.}

El $\mathrm{R}^{2}$, se mide en porcentaje, y nos indica en qué porcentaje se incrementó o disminuyó el rendimiento (variable dependiente), por cada cambio único de la(s) variable(s) independiente(s). Mientras más alto es el valor de $\mathrm{R}^{2}$, mejor es el ajuste o asociación de las variables independientes versus la variable dependiente de la línea de regresión lineal: Y= a $+b x$.

En esta investigación el $12 \%$ de incremento en el rendimiento de maní fue debido al mayor porcentaje de grano y el $20 \%$ de reducción por los valores más altos del porcentaje de vaneamiento (vainas sin grano) (Cuadro $\left.\mathrm{N}^{\circ} 2\right)$.

\section{Conclusiones.}

Una vez realizado los diferentes análisis estadísticos y agronómicos, se sintetizan las siguientes conclusiones:

- La respuesta del germoplasma de maní en cuanto a los componentes del rendimiento evaluado en esta zona a agroecológica fueron muy diferentes, con excepción del porcentaje del Vaneamiento y Semillas por Vainas. 
- Los rendimientos promedios más altos de maní, se registraron en los tratamientos T6: Pedro Carbo con 5.456 y el T13 (RCM - 112) con $5.053 \mathrm{~kg} / \mathrm{ha}$ al $14 \%$ de humedad.

- El componente del rendimiento que contribuyó a incrementar el rendimiento de maní fue el porcentaje de grano con el $12 \%$.

- El mayor número de vainas por planta se evaluó en el T8 (Perla de saavedra), con 20 vainas.

- El porcentaje de grano (PG) más alto, se registró en el T5 (Boliche), con el 88,33 \% debido a la calidad de vaina y grano.

- Las líneas seleccionadas para continuar con el proceso de investigación en este rubro en la zona agroecológica de Caluma son: T6: Pedro Carbo, T13: RCM - 112, T4 PI26202301 - 5 D y T12 SPZ - 457

- Finalmente, esta investigación contribuyó a generar información y resultados valiosos para mejorar la producción de maní en la zona agroecológica de la Granja el Triunfo, Caluma.

\section{Referencias bibliográficas.}

1.Bogado, E. 2006. Cultivo del maní. Centro Regional de Investigación Agrícola Capitán Miranda, Itapúa. Edición: Transferencia de Tecnología.

2. Caballo, A. 2005. Sanidad de semillas. Biglia Impresores. Edición $\mathrm{N}^{0}$ 6. Cordoba, Argentina. P. 103.

3. Carrillo, R. et, al. 2008. Tecnologías disponibles, para arroz, maíz, maní, caupí y yuca. Núcleo de transferencia y Comunicación. "Estación Experimental Portoviejo". Boletín divulgativo $\mathrm{N}^{\circ}$ 132. Pp.21- 26.

4.ENCICLOPEDIA AGROPECUARIA TERRANOVA. 2000. Producción Agrícola. Tomo 1. Bogotá, Colombia. Pp.152-153.

5.Espinoza, K. 2008. Módulo de Cultivos Tropicales. Guaranda, Ecuador. P.1

6.FAO. 2000. Organización de las Naciones Unidas para la Agricultura y la Alimentación, IT. 26 Conferencia Regional de la FAO para América Latina y el Caribe: Utilización de los Recursos Fitogenéticos. Mérida, MX. Pp.23-36. 
7.Fernandez, L. y Giaytto, O. 2006. El cultivo de mani en cordoba. Primera Edicion. Universidad internacional de rio Cuarto. Departamento de impreta y publicaciones de la UNRC. Rio Cuarto, Argentina. P. 186

8. Figueroa, C. 2011. Evaluación de 46 líneas de maní (arachis hipogaea 1.) de varios grupos comerciales en las estaciones experimentales litoral sur y Portoviejo. Universidad Técnica de Manabí. tesis de grado. Previo a la obtención del título de: Ingeniero Agrónomo. Manabí-Ecuador. P. 14.

9. Guillier, P. y Silvestre. 2000. El cacahuete o maní: Origen e Historia. 1ed. Barcelona, España. ES. Blume. Pp.1-9.

10. Guamán, R. y Peralta, L. 1999. Guía para el Cultivo de Maní en las provincias de Loja y El Oro. INIAP Estación Experimental Boliche (EC). Boletín Divulgativo $\mathrm{N}^{\circ} 225$. P.22.

11. INFOAGRO. 2007. El cultivo del cacahuate. San José, Costa Rica

12. INIAP. 2004. Boletín Divulgativo. Guía del cultivo para las zonas de Loja y El Oro. $\mathrm{N}^{\mathrm{o}}$. 314. Guayaquil. Ecuador.

13. Keller, M. 2009. Dirección Nacional de Agroindustria. Sector Manisero. Ministerio de Agricultura Ganadería Acuacultura y Pesca. Buenos Aires, Argentina.

14. Lavia, G. Fernández, A. 1999. Caracterización cromosómica de germoplasma de Maníes silvestre pertenecientes a las secciones Extranervosae, Heteranthae y Arachis. Cordoba, Argentina.

15. Mendoza, H. 2009. Evaluación de materiales de maní (Arachis hypogaea L.), tipo Valencia para consumo directo en la zona de Taura, Provincia del Guayas. Tesis de grado Ing. Agr. Universidad Agraria del Ecuador, Facultad de Ciencias Agrarias. Milagro, Ecuador. P.32.

16. Mendoza, H. et, al. 2005. Principal Desarrollo de variedades de maní para zonas semiseca. Est. Exp. Portoviejo. Programa de Horticultura. Informe Técnico Anual. Portoviejo, Ecuador. P. 8.

17. Monar, C. 200. Monar, C. 2000. Informe anual de actividades. UVTT-B. INIAP. Guaranda, Ecuador. P. 26.

18. Monar, C. 2010. Proyecto de investigación y Producción de Semillas. Universidad Estatal de Bolívar. Guaranda, Ecuador. P. 27 
19. Pedelini, R. 2008. Maní. Guía práctica para su cultivo. Boletín de divulgación técnica. $\mathrm{N}^{0}$ 2. INTA. EEA. Manfredi. Córdoba, Argentina. P. 2.

20. Posada, S. y Óscar, J. 2005. Fundamentos para el establecimiento de pasturas y cultivos forrajeros. Editorial Universidad de Antioquia. Colombia. P.29.

21. Ullaury, J. et, al. 2004. Guía del Cultivo de Maní para las Zonas de Loja y El Oro. EELS. Boletín Divulgativo No 314. P. 2.

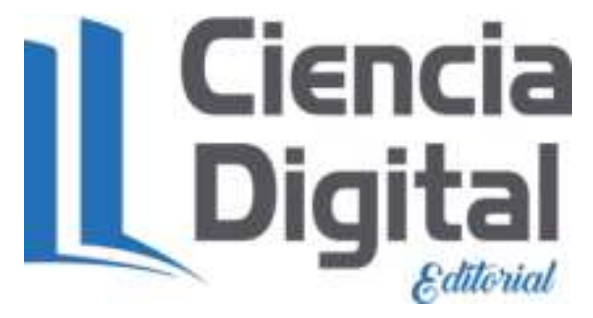




\section{Para citar el artículo indexado.}

Ibáñez S., Alcívar E., Cedeño L. \& Caicedo J. (2018). Evaluación del comportamiento agronómico de 15 líneas de maní del grupo valencia (arachis hypogaea 1.). Revista electrónica Ciencia Digital 2(1), 250-268. Recuperado desde:

http://www.cienciadigital.org/revistascienciadigital2/index.php/CienciaDigital/article/view/ $18 / 18$

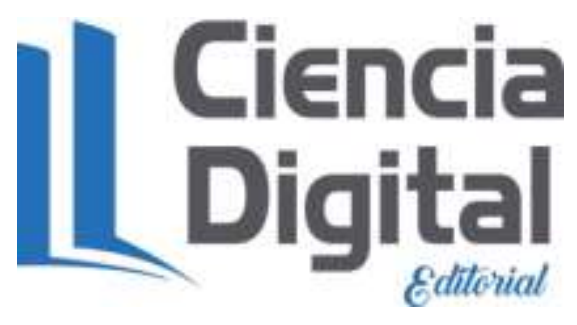

El artículo que se publica es de exclusiva responsabilidad de los autores y no necesariamente reflejan el pensamiento de la Revista Ciencia Digital.

El articulo queda en propiedad de la revista y, por tanto, su publicación parcial y/o total en otro medio tiene que ser autorizado por el director de la Revista Ciencia Digital.
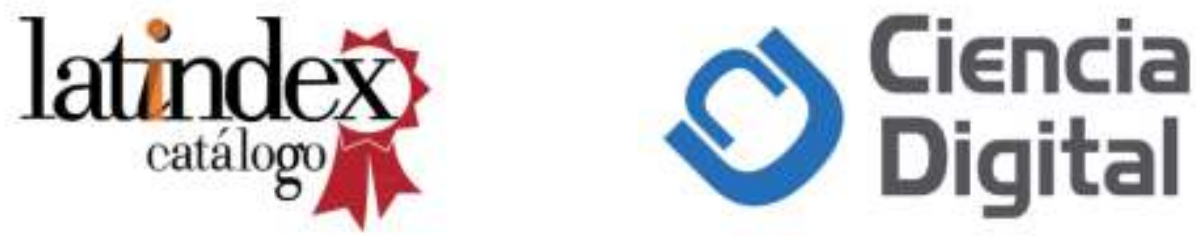